\title{
Supernova Remnants in the Magellanic Clouds
}

\author{
R. Petre
}

\author{
NASA Goddard Space Flight Center, Greenbelt, MD 20771, USA
}

\begin{abstract}
Over the past decade, substantial progress has been made in understanding the properties of Magellanic Cloud supernova remnants and their role in the ISM. Among the notable results are the "typing" of progenitors via the X-ray spectra of their remnants, the use of X-ray spectra to measure ISM abundances, and the discovery of remnants with unique properties. I summarize recent studies of MC SNRs, and describe how a refined understanding of the $S N R$ population requires consideration of the unique attributes of each remnant.
\end{abstract}

\section{Introduction}

Supernova remnants (SNRs) play a key role in the evolution of the Magellanic Clouds, as they do in all galaxies. Their enormous explosive energy, the manifestation of the gravitational collapse of their progenitor stars, is one of the primary sources of kinetic energy within a galaxy. It helps to shape the interstellar medium (ISM) and possibly catalyze the birth of a new generation of stars. The nucleosynthesized material they produce and disperse seed subsequent generations of stars (and planets) with metals. Their shocks are responsible for accelerating the cosmic rays that permeate interstellar space.

The Magellanic Clouds have long been touted as "ideal" locations for studying SNRs, both individually and collectively, and their global effect on the ISM. Many of the difficulties associated with studying Galactic remnants are overcome: the remnants are at a uniform, well-established distance, and the interstellar extinction is far lower and more uniform than in the Galactic plane. The Clouds are near enough so that detailed studies of individual remnants can be performed, especially with the latest generation of ground and space-based instrumentation. The high degree of star formation activity guarantees the presence of many massive, rapidly evolving stars, hence, supernovae. And our external view of the Clouds allow us to more easily observe the conséquences of interactions between SNRs and their surroundings. This idealistic view is tempered by reality. As we study MC remnants, we find that, like Galactic remnants, each has unique attributes. These must be taken into consideration before any credible general statement about MC SNRs can be made. 


\section{Demographics}

There has been no "census" of MC SNRs since the work of Mathewson et al. (1983, 1984, 1985). They cataloged 32 LMC SNRs and 11 SMC SNRs. Based on this catalog and subsequent discoveries, the numbers of confirmed LMC and SMC SNRs stand at 38 and 23. The progenitor type of some is well established, while for others we can make a reasonable guess. The four LMC SNRs located in relatively gas free regions, whose optical emission consists exclusively of $\mathrm{H} \alpha$ filaments characteristic of nonradiative shocks, are thought to be remnants of Type Ia explosions. The X-ray spectra of two of these are dominated by line emission from Type Ia nucleosynthesis products, as is the spectrum of N103B. There are three O-rich remnants, N132D and N158A in the LMC and E010272 in the SMC, which most likely had massive progenitors. N158A also has a central pulsar, strengthening the massive progenitor case; a pulsar has also been recently discovered in N157B. Circumstantial evidence, i.e., proximity to $\mathrm{H}$ II regions or OB associations, indicates that at least 18 other LMC SNRs had massive progenitors (Chu \& Kennicutt 1988; Smith et al. 1994). And of course, SN1987A was a Type II SN. In contrast, no systematic study of the environments of SMC SNRs has been carried out, so with the exception of E0102-72, none of the SMC SNRs have been "typed."

Table 1. Current Census of MC SNRs.

\begin{tabular}{lcccc}
\hline & Total & Type Ia & Type II/Ib/Ic & Unknown \\
\hline LMC & 38 & 5 & $24(+1 !)$ & 8 \\
SMC & 23 & 0 & 1 & 22 \\
\hline \hline
\end{tabular}

In Table 2, we compare the morphological types of the SNRs in the Mathewson et al. catalog with those in the Galaxy. As in the Galaxy, the majority of the MC remnants are shell-like. Although the numbers are small, there is a dearth of known composite and mixed morphology remnants in the Clouds.

Table 2. Demographics of MC SNRs.

\begin{tabular}{lccc}
\hline Type & LMC & SMC & Milky Way \\
\hline & 25 & 8 & 148 \\
Shell & 2 & 1 & 9 \\
Filled & 2 & 1 & 30 \\
Composite & 1 & 0 & 14 \\
Mixed Morphology & 2 & 1 & 14 \\
Undetermined & 3 & 0 & 34 \\
\hline Central Compact Objects & 3 \\
\hline \hline
\end{tabular}

\section{Recent Discoveries - Individual Remnants}

N103B: This remnant is the best example of why care must be taken before making generalizations about MC SNRs. Based on its interaction with an $\mathrm{H}$ 
II region, it was thought to have a massive progenitor (Dickel \& Milne 1995). The X-ray spectrum, however, shows clear evidence of Type Ia nucleosythesis products (Hughes et al. 1995). A recently acquired deep ASCA spectrum of N103B, reveals a strong Fe $\mathrm{K}$ line at $\sim 6.5 \mathrm{keV}$ (Hwang et al. 1999).

E0102-72: In constrast to the X-ray spectrum of N103B is that of the O-rich SMC remnant E 0102-72 (Hayashi et al. 1993). It is dominated by strong $O$ and $\mathrm{Ne}$ lines, indicative of a massive progenitor. The $\mathrm{O}, \mathrm{Ne}$, and $\mathrm{Mg}$ lines are all produced in different ionization conditions, suggesting that the ejecta are stratified, unlike the turbulently-mixed Type II remnant Cas A. Radio observations reveal a central enhancement, which could be a plerion (Amy \& Ball 1993).

N132D: HST narrow band images of this O-rich LMC SNR resolves spatial scales comparable to ground based images of Galactic SNRs (Morse et al. 1996). Ionization structure is discerned within shocked interstellar clouds and filaments, but not within the O-rich ejecta. The X-ray emission is closely correlated with shocked clouds, but not ejecta. The ionizing radiation from the main blast wave is not sufficient to generate the optical emission from the precursor outside the rim; photons produced in slower shocks moving through clouds around the outer rim can supply sufficient additional radiation.

N157B and N49: Two important discoveries have recently been made involving central compact objects within LMC SNRs. A pulsar has been found in N157B, whose $16 \mathrm{~ms}$ period is the fastest known for a pulsar associated with a SNR (Marshall et al. 1998). N49 has long been thought to house the soft $\gamma$-ray repeater SGR 0526-66, famous for its eruption on March 5, 1979. Danner et al. (1999) report variability from the X-ray counterpart of this object, which might have a periodicity of 95 days.

There are other notable results. The complex optical morphology of N63A can be explained as the result of the interaction between the shock front and ISM features of sizes between 0.01 and $5 \mathrm{pc}$ (Chu et al. 1999). The complex structure of the Honeycomb SNR can be understood as an old shock front interacting with the dense, but porous, wall of a pre-existing cavity in the line of sight (Chu et al. 1995). The SMC remnant $0101-7226$ has a $\mathrm{X}$-ray to radio luminosity ratio at least a factor of three less than any other known SNR (Ye et al. 1995).

\section{Recent Collective Studies}

SNRs in OB Associations: Chu (1997) has performed a careful search for high velocity gas in 14 LMC SNRs positionally coincident with OB associations. She found that SNRs in H II regions all show classical SNR signatures (bright Xray emission, nonthermal radio emission and enhanced $[\mathrm{S} \mathrm{II}] / \mathrm{H} \alpha$ ratio) as well as high-velocity gas, while some SNRs in superbubbles show only X-ray emission. These differences can be traced to the environments in which the remnants are expanding. Her results suggest that some SNRs might be missed if they are classified as such only if they have all three classical signatures.

X-ray Spectral Surveys: ASCA has produced other two key results. Spectroscopy of several evolved LMC remnants has been used to infer the ISM metal abundances (Hughes et al. 1998); the results are consistent with those of Russell \& Dopita (1992). Petre et al. (1999) have searched for evidence of high energy cosmic ray acceleration in shell-like LMC remnants in the guise of nonthermal 
hard X-ray tails. Several young SNRs might have nonthermal components comparable to that of the Galactic remnant SN1006, but none is found in the older remnants in the sample. This result places the first observational limit on the age to which SNRs efficiently accelerate cosmic rays.

Radio Survey: Dickel and co-workers (e.g., Dickel \& Milne 1995) have used ATCA to measure the spectral index and produce polarization maps of approximately a dozen MC SNRs. They show that most of these appear morphologically similar to their Milky Way counterparts.

\section{Conclusions}

The abundance of data in all bands has provided an opportunity to study the MC SNRs at a level of detail comparable with many Galactic remnants. These studies have shown that the MCs possess the full range of analogs to Galactic SNRs, as well as some unique objects. All of the remnants studied show distinctive features. These studies also show that care must be taken when performing collective studies to account for distinctive properties, such as expansion into cavities and against $H$ II regions and other large structures. Not taking these into account affects inferences about ages, energy, and progenitor type. Far less attention has been paid to SMC SNRs than their LMC counterparts. Now that a reasonably sized sample of candidates exists, this situation should be remedied.

\section{References}

Amy, S.W., \& Ball, L. 1993, ApJ, 411, 761

Chu, Y.-H. 1997, AJ, 113, 1815

Chu, Y.-H., et al. 1995, AJ, 109, 1729

Chu, Y.-H., et al. 1999, this volume

Chu, Y.-H., \& Kennicutt, R.C. 1988, AJ, 96, 1874

Danner, R., et al. 1999, A\&A, in press

Dickel, J.R., \& Milne, D.K. 1995, AJ, 109, 200

Hayashi, I., et al. 1993, PASJ, 46, L121

Hughes, J.P., et al. 1995, ApJ, 444, L81

Hughes, J.P., et al. 1998, ApJ, 505, 732

Hwang, U., et al. 1999, this volume

Marshall, F.E., et al. 1998, ApJ, 499, L179

Mathewson, D.A., et al. 1983, ApJS, 51, 345

Mathewson, D.A., et al. 1984, ApJS, 55, 189

Mathewson, D.A., et al. 1985, ApJS, 58, 197

Morse, J.A., et al. 1996, AJ, 112, 509

Petre, R., et al. 1999, ApJ, submitted

Russell, S., \& Dopita, M.A. 1992, ApJ, 384, 508

Smith, R.C., et al. 1994, AJ, 108, 1266

Ye, T., et al. 1995, MNRAS, 275, 1218 